\title{
Assessment of stone heritage decay by X-ray computed microtomography: I - a case study of Portuguese Braga granite
}

\author{
Maurício, A. , Figueiredo, C. , Pereira, M.F., Alves, C. ${ }^{* *}$, Bergounioux, M. ${ }^{* * *}$ and Rozenbaum O. ${ }^{* * *}$
}

* CERENA/CEPGIST, Instituto Superior Técnico, Universidade de Lisboa, Lisbon, PORTUGAL

***entro de Investigação Geológica, Ordenamento e Valorização de Recursos - Universidade do Minho, PORTUGAL

****Université d'Orléans, UFR Sciences, Math., Labo. MAPMO, UMR 6628, Route de Chartres, BP 6759, 45067 Orléans cedex 2, FRANCE

*****Institut des Sciences de la Terre d'Orléans (ISTO) UMR7327 CNRS/Université d'Orléans, FRANCE

Email : pcd2045@ ist.utl.pt

Within a context of heritage decay following previous studies [1] [2], this study aims to validly map, model and segment 3D mineral matrix and/or voids spatial structures (textures) of Portuguese Braga granite, using 3D microscopy image analysis. Texture properties of the geologic materials play a dominant role in their petrophysical and petrochemical properties. These petrographic properties can be non-destructively mapped and/or further studied with the use of quantitative $\mu$-XCT imaging and its parameterization as well. $\mu$-XCT image sets are analogous to images that would be obtained more tediously and laboriously with serial sectioning. Furthermore, because the images are digital, the method lends itself more easily to both quantitative analysis and widespread dissemination [3]. The samples were scanned with an industrial CT device Nanotom 180NF, using a $3 \mu \mathrm{m}$ pixel size resolution. Since granite is a multicomponent, multiphasic, random-heterogeneous natural more or less porous material, a fundamental step that must be considered after carefully controlling the quality of the scanning, reconstructing and rendering processes is the image segmentation process of its component phases. Expert-valid segmentation of these images into minerals and/or voids subspaces is critical to the integrity and accuracy of geomaterials modal/textures' studies. The absence of a single image-processing algorithm to provide such an accurate separability, immune to all the intricacies of the acquired scanning, makes this seemingly simple task significantly error prone and almost certainly case-study dependent. Consequently, this research methodology aims to adaptively select a better segmentation procedure for this case study. This can be performed guided by a criterion selecting the more robust, easy-to-implement, unambiguous, signal-processing-based, segmentation procedure. Specifically, representative digital photographs of the same granite type, in two conditions, sound and mildly weathered at the same scale resolution, are compared with the results of the two different algorithmic procedures, then, ranking them and chose the best (Figs. 1-2). This enables at least expert-visual-correlating and ranking the segmented images and the digital photographs, qualitatively evaluating and validating the algorithmic procedure in this case study. With the use of this methodology it is possible to segment the reconstructed and 3D rendered images of a sound Portuguese Granite and choose between the two constructed segmentation procedures. The proposed solution for this case study has been extensively tested on $\mu$-XCT scanned images of two samples of sound and weathered granites. The suggested methodological approach and the results obtained may have implications and developments are expected for more accurate image-based characterization of magmatic geomaterials composing heritage objects. Further critical examination follows in a more extended article where a qualitative/quantitative parameterization assessment and statistical 
validation will be the next step to be taken on these case studies. Although Procedure 2 is clearly better than Procedure 1, it didn't allow to segment feldspars (gray). However, ferromagnesians (green), quartz (yellow) and opaque minerals (red) are clearly segmented. This study, demonstrates the applicability potential of micro-tomography in providing new insights and guidance to prepare more systematic and detailed petrographic investigations, within the context of stone heritage, enabling integrated research for more rational design and control it's composing geological materials decay. This is because of $\mu$-XCT relatively easy 3-D information visualisation and quantification potential and non-destructive character.

The authors acknowledge FCT projects: PEst-OE/CTE/UI0098/2011 and PEst- OE/CTE/UI0697/2011.

[1] Y. Géraud, et al., Geological Society, London, Special Publications, 215, 95-105, 2003.

[2] J.M.S. Matias, et al., Geological Society, London, Special Publications, 205, 273-281, 2002.

[3] R.A. Ketcham, et al., Computer \& Geosciences, 27, 381-400, 2001.
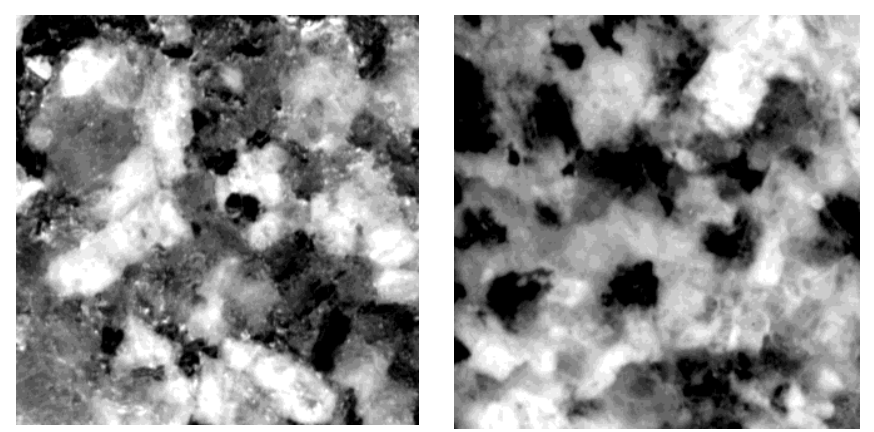

Figure 1 - Representative digital scanning photographs of mildly weathered (Left) and sound (Right) granite surfaces. Square sizes 5x5mm². White - Feldspar; Grey - Quartz/Feldspar; Black - Biotite and Ilmenite.

Procedure 1: Perspective views of part rendered and segmented core sample.
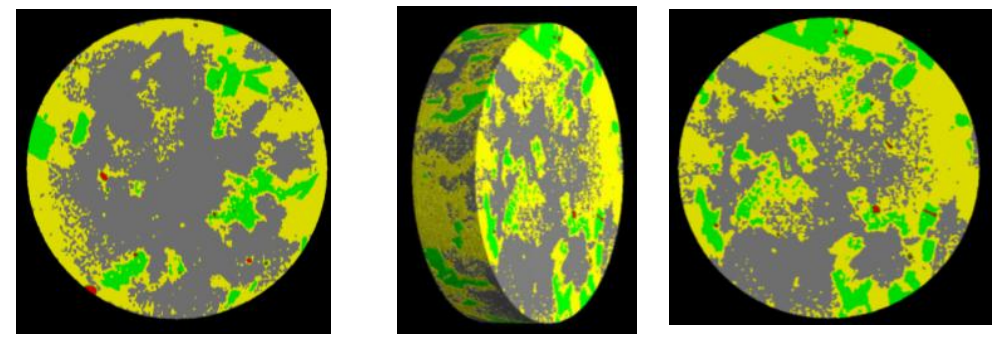

Procedure 2: Perspective views of part of rendered and segmented core sample.
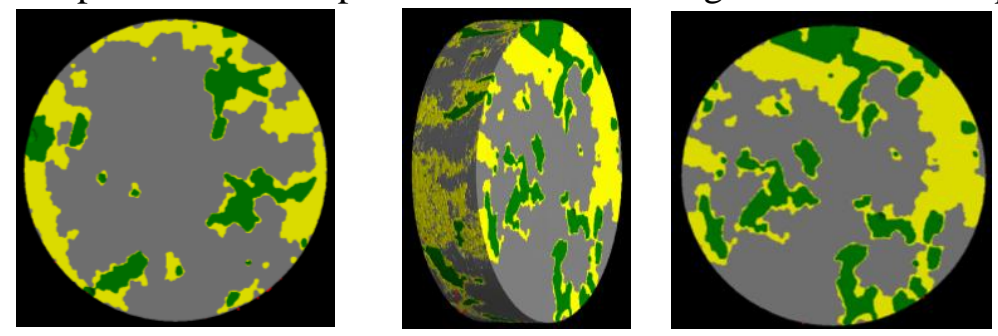

Figure 2 -Two sets of three perspectives of the rendering-segmentation procedure results corresponding to Procedure 1 and Procedure 2. (Core sample diameter scales 5mm). Gray - Feldspars; Yellow - quartz; Green - Biotite; Red - Ilmenite. A valid ranking step is possible, comparing Procedure 1 and Procedure 2 and Fig.1. 